\title{
Aplicación de la guadua para la construcción de cubiertas con tierra, basadas en el "domocaña"
}

\section{Application of the guadua for the construction of roofs with earth, based on the "domocaña"}

\author{
Clara Ángel Ospina* y Lucía Esperanza Garzón***
}

Citar este artículo como: Ángel, Clara y Garzón, Lucía (2017). Aplicación de la guadua para la construcción de cubiertas con tierra, basadas en el "domocaña”. Revista Nodo, 12(23), 49-61

\section{Resumen}

Este artículo describe el trabajo experimental de construcción de varias viviendas realizadas con la técnica de cubierta de guadua y tierra denominada "domocaña", innovación realizada en la Universidad Nacional de Ingeniería del Perú. Esta es una transferencia reciente, solo lleva cerca de una década de aplicación en Colombia; existen varias obras experimentales y demostrativas que fueron construidas con esta técnica, por profesionales que aceptan el desafío de la innovación para el desarrollo de conocimiento. Se considera que esta propuesta técnica tiene validez y amerita un trabajo más profundo, puesto que las cubiertas que se han logrado construir son alternativas versátiles, ecológicas, durables, bonitas, confortables climática y acústicamente, económicas y sencillas de elaborar.

Este escrito propone estimular el estudio de dicha técnica desde la descripción de las experiencias para trasferir lo aprendido a partir de la práctica constructiva, con los aciertos y las dificultades. Todo ello en virtud de que para cubiertas de viviendas de bajo costo es una alternativa que tiene un alto potencial; de ahí que la intención sea motivar a otros profesionales e investigadores, incluso a centros de estudios como las universidades, para que profundicen científicamente en esta propuesta de construcción.

Palabras clave: Cubierta con guadua y tierra, domocaña, techo ecológico.

\section{Abstract}

This article describes the experimental work of construction of several houses made with the guadua and land cover technique called "domocaña", innovation carried out at the National Engineering University of Peru. This is a recent transfer, it only takes about a decade of application in Colombia; There are several experimental and demonstrative works that were built with this technique, by professionals who accept the challenge of innovation for knowledge development. This technical proposal is considered to be valid and merits deeper work, since the roofs that have been built are versatile, ecological, durable, beautiful, climate and acoustically comfortable, economical and simple to develop alternatives.

Fecha de recepción: 22-05-2017 • Fecha de aceptación: 20-10-2017

\footnotetext{
* Arquitecta. Correo electónico: claraangelospina@gmail.com

** Arquitecta independiente. Correo electónico: luciagarzon@gmail.com
} 
This paper proposes to stimulate the study of this technique from the description of the experiences to transfer what has been learned from the constructive practice, with the successes and difficulties. All this by virtue of which for low-cost housing covers is an alternative that has a high potential; hence the intention is to motivate other professionals and researchers, including study centers such as universities, to deepen scientifically in this construction proposal.

Keywords: Cover with guadua and earth, domocaña, ecological roof.

\section{Introducción}

En la construcción de cualquier obra habitacional una de las actividades que consume entre un 25 a un 30\% de los recursos económicos es la cobertura. En la vivienda social este ítem es considerable y son pocas las alternativas que hay en el mercado para responder a las exigencias que debe cumplir este elemento constructivo. En el caso de los países tropicales, con vulnerabilidad sísmica y con diversos climas y condiciones ambientales, los techos deben adaptarse a todos estos requerimientos.

La necesidad de cubrir grandes luces con menores espesores fue un avance que se presentó con los materiales industriales que inspirados en las formas esféricas pueden soportar dichas cargas. El principio es aprovechar la "forma como estructura”, que absorbe los esfuerzos de compresión y tracción y que desencadenó posteriormente en nuevas posibilidades formales.

En esta búsqueda surgen los cascarones de concreto que hacen parte de las estructuras laminares, técnica que surgió de la geometría y nace a finales del siglo XIX, a partir de la era industrial y que, desde el siglo XX, desarrolló una tipología amplia de estructuras con hormigón armado.

El procedimiento de construir las cáscaras con estructuras resistentes por la forma-requiere de una formaleta o cimbra para su montaje; después se realiza el armado con el hierro para recibir el hormigonado con una mezcla proyectada y dejar que fragüe, para al final desencofrar y, de esta manera, obtener los cascarones, dando la "forma" al material según lo que necesitan soportar. Como las cargas son pequeñas, las formaletas o cimbras son también fáciles de montar.

De la técnica de cascarones de concreto surgieron múltiples formas, entre ellas las cúpulas, láminas cilíndricas como bóvedas, placas plegables, hiperboloides y paraboloides hiperbólicos, que presentan una curvatura convexa y cóncava, incluso aparecen las cimbras hinchables, entre otras. Esta evolución técnica, como consecuencia de los nuevos materiales de la era industrial y de las propiedades del cemento y el acero, fue la que impulsó el desarrollo tecnológico con otras posibilidades, como los elementos prefabricados, cascarones de hormigón armado el uso de cables como refuerzos y los pretensados.

La mayoría de las cúpulas tiene forma circular, aunque hay algunos ejemplos elípticos. Todas se deben diseñar para resistir los empujes horizontales, de otro modo generan esfuerzos que se expandirán al producir tensión perimetral. Las cúpulas elípticas se definen por la rotación de media elipse alrededor de su eje vertical; es menos eficiente que una cúpula esférica, pues la parte superior de la cáscara es más plana y la disminución de curvatura introduce mayores tensiones. Estas posibilidades tecnológicas abrieron paso a otras formas de expresión y lenguaje arquitectónico en el siglo pasado.

Con estos antecedentes técnicos e industriales, surge la idea del domocaña. Haciendo una extrapolación de los materiales, pensando en lo social y sostenible, una vez que los cascarones funcionan con materiales industrializados, ¿por qué no ensayar con materiales no convencionales? El empleo de las bambusas en funciones similares de la estructura y el remplazo del concreto por la tierra estabilizada, fue la inspiración de esta investigación que se realizó en la Universidad Nacional de Ingeniería del Perú (UNI), explorando otras soluciones de cubiertas para los sectores de menos recursos. 
La búsqueda de un techo económico que utilice recursos locales, de fácil construcción y sismo resistente llevó a proponer el uso de la caña (carrizo, caña brava o bambú), como material constructivo por sus excelentes características de resistencia y por ser un material conocido y usado en la fabricación de esteras que es una lámina tejida de caña trenzada muy usada en Suramérica y en Perú. En estas experimentaciones se usó malla gallinero, revestimiento de mortero cemento-arena o barro, con características de seguridad, habitabilidad y bajo costo.

De esta manera, por encargo de la Comisión del Ministerio de Vivienda, Construcción y Saneamiento del Perú, y con el fin de dar la norma técnica, aun se está estudiando las características físicas y mecánicas del material para establecer parámetros de diseño y condiciones constructivas para el uso de la caña y bambú en edificaciones. En ese sentido, con el respaldo de la investigación académica, se realizaron ensayos de cargas estáticas en el Laboratorio del Centro de Investigaciones Sísmicas y Mitigación de Desastres (CISMID-UNI) en Perú y construcciones con techos de domocaña resistieron cargas dinámicas en los sismos de Moquegua (2003) y Pisco (2007) sin sufrir daño alguno. Dicha iniciativa investigativa se orientó también a lograr un proceso constructivo de fácil aprendizaje para ser aplicado por la comunidad beneficiaria o por microempresas.

El proceso de retorno a las técnicas vernáculas a través de esta innovación, dio inicio a otra posibilidad de cobertura que parte del referente del bahareque de guadua (caña) y tierra, aplicado en paredes y traspasado a cubiertas ahora en Colombia.

\section{Descripción de la técnica y experiencias}

El domocaña es una cubierta de forma geométrica orgánica, tipo cúpula o bóveda, con estructura de material vegetal recubierto con mortero de tierra, cal o cemento. Podría decirse que es una cubierta catalogada en la construcción con tierra como una técnica mixta.

Una de las primeras experiencias previas con materiales industriales fue presentada en el proyecto del Cyted XIV.5 con techo y el 10 x 10 realizado en Chile, con la solución de un techo tipo cúpula de ferrocemento, desarrollado por la Universidad Autónoma de México. Posteriormente como producto de la investigación e innovación, la UNI de Perú realizó un domocaña con materiales naturales, propuesta que ha sido difundida en múltiples talleres con reconocimientos internacionales. Un equipo de profesionales dentro de FAUA- UNI, contribuyó con esta y otras investigaciones al avance y la difusión de la construcción con tierra y de otros materiales en el resto del mundo, participando en actividades de transferencia tecnológica en varios continentes.

De la herencia de los cascarones de concreto, que fue la técnica originaria, se traspasó a un "cascarón de domocaña". La proyección se ha realizado para obras más simples y domésticas, por ser más accesible y usar materiales locales y naturales como resultado de la innovación; también por ser más fácil de construir y no requerir de formaleta $o$ cimbra como sí lo exige el cascarón de concreto. Además usa la forma como estructura que en este caso se realiza con las tiras de guadua cortadas y flexionadas de forma natural, con dimensiones proporcionales a la luz a cubrir, constituyéndose en una manera de velar espacios de forma económica y ecológica.

Según Barrionuevo (2001), la motivación fue desarrollar una cubierta accesible para la gente más necesitada y resalta que "en el Perú, al igual de otros países en vías de desarrollo, existe la 'urgente necesidad de atender la cada vez más creciente demanda de vivienda', en especial de los marginados" (p. 52). Además, estos grupos tratan de resolver su problema de morada autoconstruyendo en forma progresiva su vivienda, comenzado con los muros y se detienen en el techo porque es un proceso difícil y de costo muy alto. En ese sentido el miso autor señala que la 

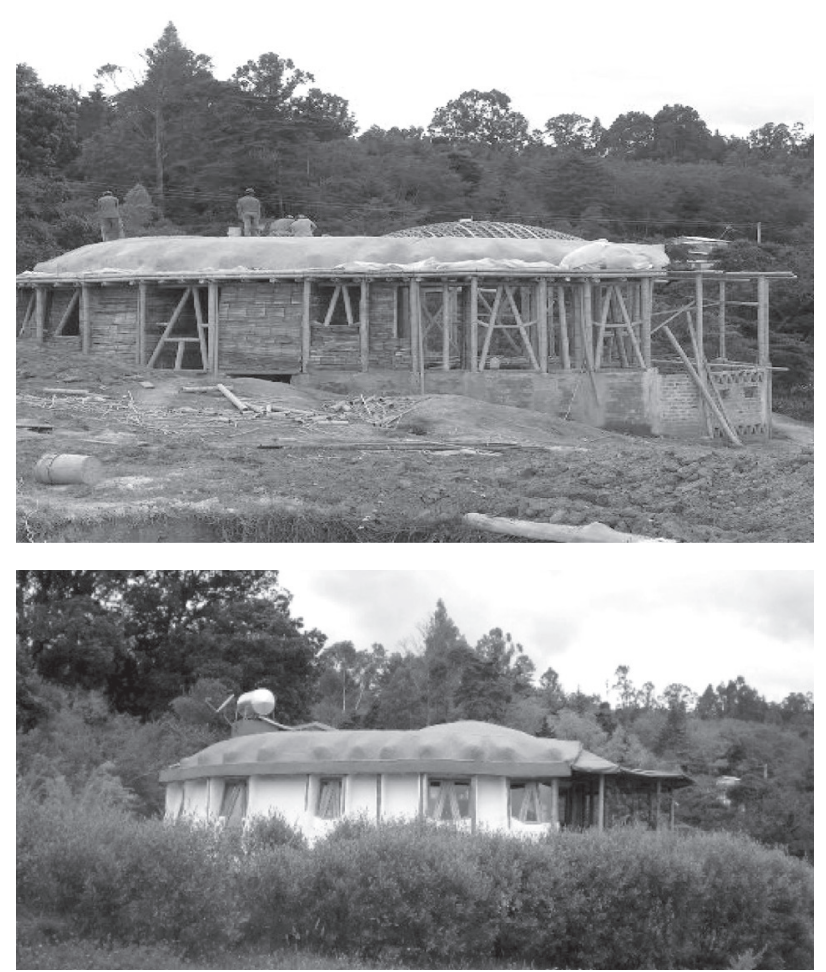

Figura 1. Proceso de la casa en bahareque y cubierta en domocaña Reserva Natural Cochahuaira, Gachantivá, Boyacá. Proyecto y construcción: arq. Clara Ángel. Fuente: propia

caña por ser "un recurso natural renovable" y tener unas "características mecánicas y físicas, las ventajosas posibilidades que significan su uso en la construcción de viviendas y por su capacidad de reducir el $\mathrm{CO}_{2}$ " (p. 52), explora en otras soluciones para crear una vivienda sostenible y por ello se plantea el estudio de un techo de bajo costo, usando recursos locales, de fácil proceso constructivo y sismo resistentes.

Las gramíneas como el carrizo, caña brava, caña de Guayaquil o bambú, que incluye la guadua, son de fácil y rápido crecimiento (esta última en siete años apróximadamente), se desarrollan especialmente en las orillas de las fuentes de agua y en las zonas tropicales; por sus excelentes características de resistencia, han sido usadas históricamente por familias de escasos recursos.

Con estos antecedentes se construyó el primer domocaña en Colombia, adaptando materiales locales como la guadua, y producto del taller
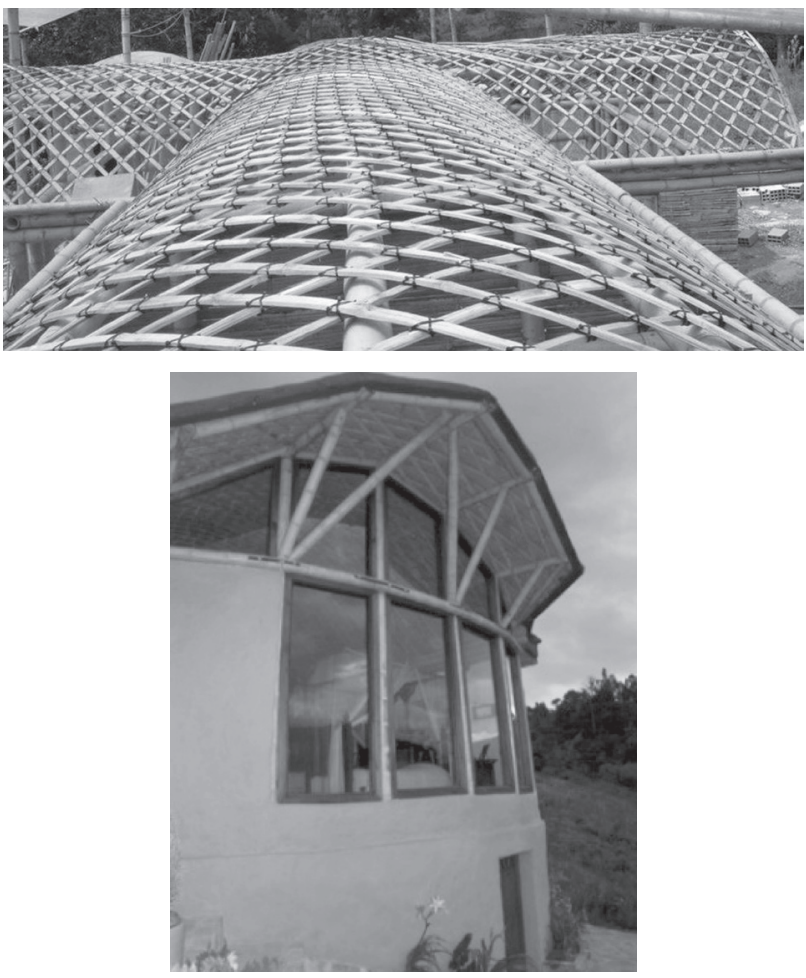

Figura 2. Evolución y formas de domocañas construidos en Villa de Leyva y en la Vega Cundinamarca. Fuente: propia

de transferencia de tecnología. El techo de domocaña, cúpula de tierra, fue inspirado en el aprendizaje realizado en San Salvador dentro del SICOT -Seminario Internacional Construcción con Tierra- promovido por el proyecto Proterra / Cyted y organizado por la Fundación Salvadoreña de Desarrollo y Vivienda Mínima (FUNDASAL, 2004).

En Colombia se inicia este proceso de adaptación y asimilación tecnológica, cambiando las cañas bravas por tiras o "latas" de guadua, que resultan aptas para aplicar en esta cubierta, por ser un material con mayor longitud y tener otras propiedades estructurales.

\section{Un recurso renovable: la guadua}

La guadua Angustifolia Kunth es una gramínea gigante de América, nativa de Colombia, Venezuela y Ecuador. Esta especie ha sido seleccionada como 
uno de los mejores bambúes del mundo debido a sus excelentes propiedades físico-mecánicas y es considerada como la más importante especie nativa de Colombia. En nuestro país se encuentra principalmente en la cordillera central, en suelos con características volcánicas, en las zonas de ladera y en las riberas de los ríos y quebradas. Esta especie hace parte del desarrollo cultural, económico y de conservación de los recursos hídricos en países de América Latina. Sus cualidades físicas, su bajo costo y disponibilidad la convierten en un material ideal para uso en la construcción de viviendas para familias de escasos recursos económicos, que también la pueden utilizar para otros usos como la elaboración de muebles, instrumentos musicales e incluso como leña. Esta guadua tiene diversas proporciones y comercialmente se vende inmunizada y seca con largos entre seis y ocho metros y un diámetro entre $10 \mathrm{~cm}$ y $12 \mathrm{~cm}$. (madura).

En ciertas regiones como la zona cafetera se encuentran guaduas hasta de 50 metros de largo, brindando la posibilidad de realizar mayores luces en cubiertas, si se construye en el sitio donde se recolecta el material. Colombia cuenta con abundante insumo y mano de obra calificada para la construcción con guadua. Es necesario demostrar nuevas aplicaciones y romper el prejuicio que se tiene con este material.

Durante las últimas décadas han aumentado las áreas sembradas dentro de cadenas sostenibles y hay empresas dedicadas al cultivo, procesamiento y comercialización para su uso en la construcción; igualmente entidades como el Servicio Nacional de Aprendizaje (SENA) y otros centros de formación, cualifican el personal técnico.

Aunque existen construcciones de gran calidad y diseñadores con reconocimiento internacional que promueven el manejo de la guadua, la técnica de domocaña todavía no está difundida, ni se ha popularizado. En esta técnica la guadua es usada como material para la estructura de la cubierta, la cual se construye como un tejido o malla firme y resistente; ésta debe de fijarse con una prensa $\mathrm{y}$ apoyarse en la viga perimetral, elemento que
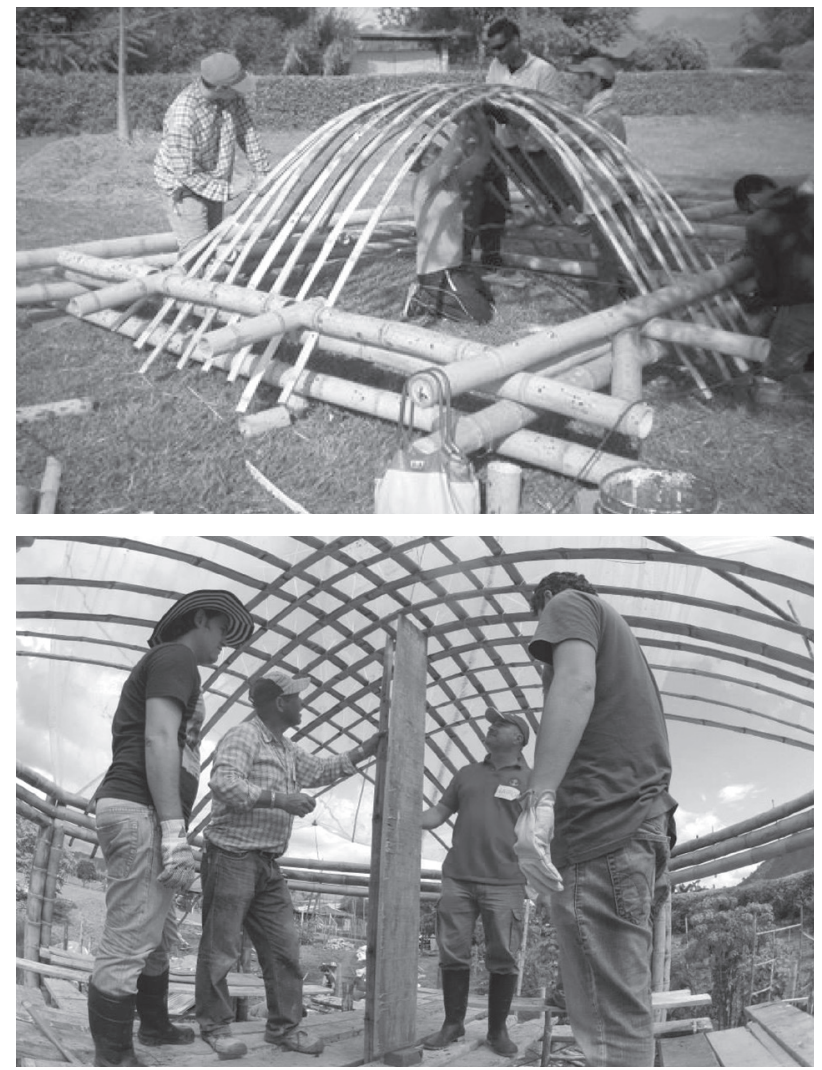

Figura 3. Elaboración de la trama de "latas" de guadua en domocaña, construidas prefabricadas desde abajo para montarla a las estructuras o en el lugar desde el techo. Fuente: propia

sostiene los arcos que se conforman con las tiras a flexión.

De una pieza de bambú normalmente pueden salir entre seis a ocho tiras también denominadas "latas", con dimensiones de una pulgada de ancho aproximadamente y con un grosor de un centímetro; al colocarla a flexión-propiedad del mismo material, da una curva limite que funciona muy bien para proporcionar la forma en arco.

La construcción del domocaña se puede realizar de varias formas, dependiendo del diseño y el tamaño. Una de ellas es desde abajo, como un prefabricado; para ello primero se teje en el piso y se pone encima de las paredes o estructura para después colocarle el revestimiento encima. La otra es construir e instalar arriba, forma que requiere de andamios para tejer la cubierta sobre las mismas 
paredes, con las debidas normas de seguridad por ser trabajo en altura.

Para elaborar la trama se utilizan guaduas maduras y secas, trabajadas con las tiras denominadas localmente "latas", estas tienen una sección longitudinal, nunca se deben añadir o traslapar; se cortan de anchos variables, y la distancia entre ellas debe estar definida previamente de acuerdo a la luz a cubrir. Las proporciones como la distancia entre latas oscila entre 15 y $20 \mathrm{~cm}$., depende del peso a soportar y los esfuerzos a los que se van a someter las estructuras, así como la forma, el espacio y el diseño que debe ser el resultado una propuesta estética.

Por la experiencia en las obras realizadas se puede decir que este tipo de techo tiene un costo económico realmente más bajo que otras técnicas del mercado; la mano de obra es muy simple, sólo requiere de una capacitación básica pues con una mínima instrucción cualquier persona puede montar y hacer el entramado. Es necesario, eso sí, tener en cuenta las dimensiones y la geometría respectiva para lo cual es pertinente una asesoría técnica.

Si se compara con otras cubiertas el costo ambiental también es bajo, consume poca energía y material. A manera de ejemplo, para un módulo cuadrado de $3.60 \mathrm{~m} \times 3.60 \mathrm{~m}$ se puede hacer la trama de la cubierta con seis u ocho guaduas cortadas en tiras, lo cual es suficiente para cubrir dicha superficie.

Se debe tener en cuenta que esta técnica exige tener muy estable la estructura del anillo donde se apoya la malla. El anillo periférico puede construirse con material convencional (hormigón armado), madera o con guadua.

El entramado curvo debe fusionarse con el anillo conformado por la viga de amarre y, sobre este anillo estructural que soporta toda la cubierta, se aprieta con una prensa que puede ser de la misma guadua, o caña; previamente se debe proyectar el empalme entre la malla o tejido y la estructura de soporte del anillo superior debe resistir el empuje lateral.
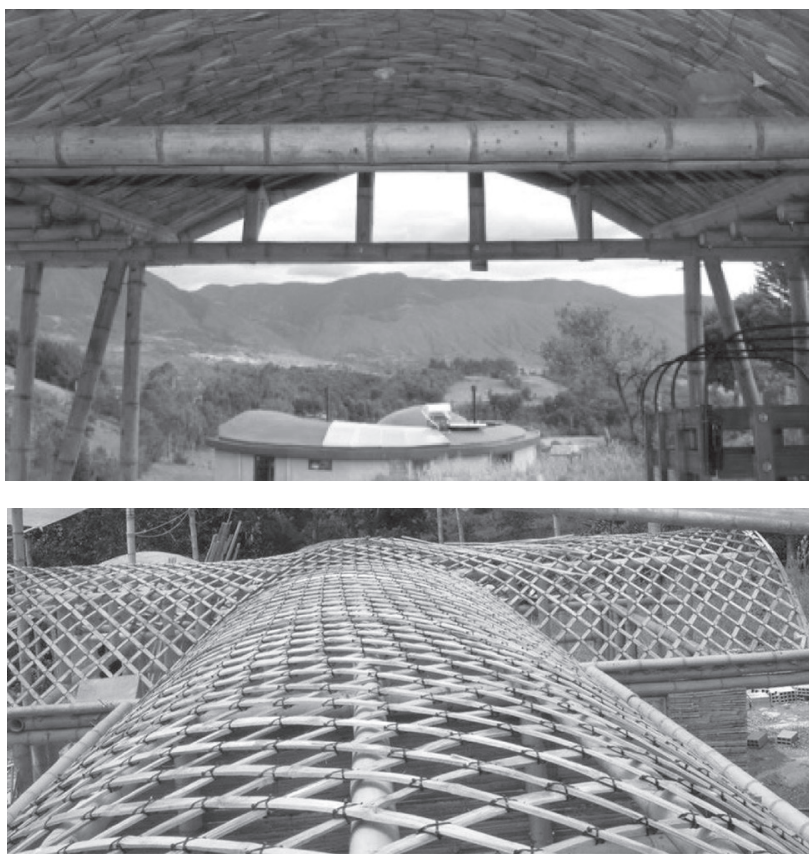

Figura 4. Diferentes posibilidades estructurales del domocaña: a) con esterilla de guadua; b) con latas de guadua. Fuente: propia

Con las latas se elabora la estructura de la trama con diferentes posibilidades formales, la superficie se teje como una malla o cuadrícula, dependiendo de la forma: cúpula o bóveda. Se puede amarrar las latas con alambre, aunque se facilita mas con tiras plásticas denominadas "zunchos", colocadas en forma de cruz. Los zunchos plásticos son usados generalmente para las instalaciones eléctricas y este recurso resultá más ágil de amarrar que el alambre. La función que cumplen estos amarres es la misma de los hierros en la estructura de concreto, ya que la totalidad de ésta debe funcionar como la cestería: flexible pero resistente.

La dirección de la colocación de la malla o retícula se hace de acuerdo a la forma de la construcción. Si la planta de la cubierta es cuadrada, se colocan en forma de cuadricula y con la misma dirección de las vigas de amarre; si es en forma abovedada se colocan diagonal a las vigas. Es muy importante la forma de ensamblar estas latas a la viga de amarre, ya que de ahí depende el funcionamiento estructural y la amplitud del espacio. Es fundamental prever un sistema de desagüe de aguas lluvias. 

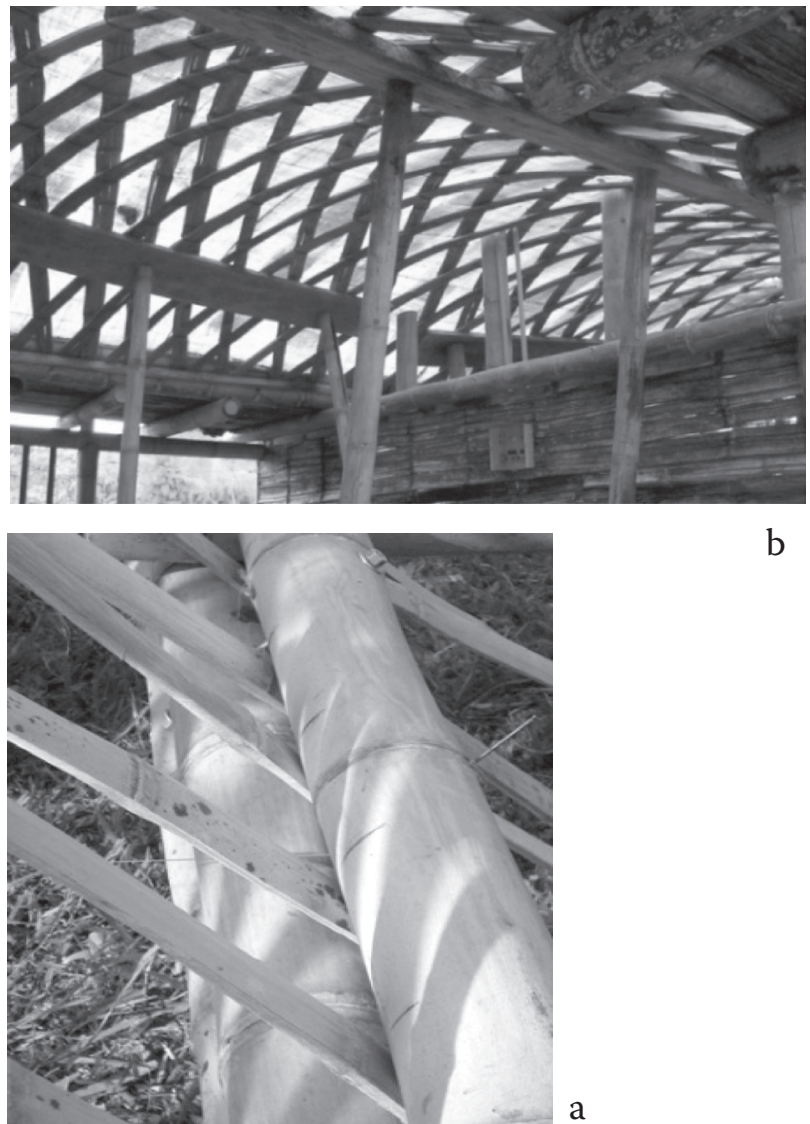

b

Figura 5. a) Apuntalamiento del domocaña para mantener la forma durante la colocación y secado del mortero. b) Un tipo de ensamble con la viga de amarre. Fuente: propia

Cuando la luz o distancia entre las paredes es mayor a tres metros se requiere además apuntalar esta red o malla desde abajo pues, dada la flexibilidad de este canasto, se puede deformar con el peso del mortero de tierra. Es importante conservar la forma curva mientras se seca el mortero, pues es la forma de arco la que garantiza su adecuado trabajo estructural a la compresión.

Una vez lista la estructura de esta malla, si se desea dejar a la vista el interior, se coloca encima de ella tela de costal tipo yute $\mathrm{u}$ otra, siguiendo la forma y cuidando el acabado; también puede pañetarse y dejar mortero al interior, protegiendo mejor la estructura vegetal.

La estructura del entramado sostiene el peso del mortero, que en un principio es pesado al estar
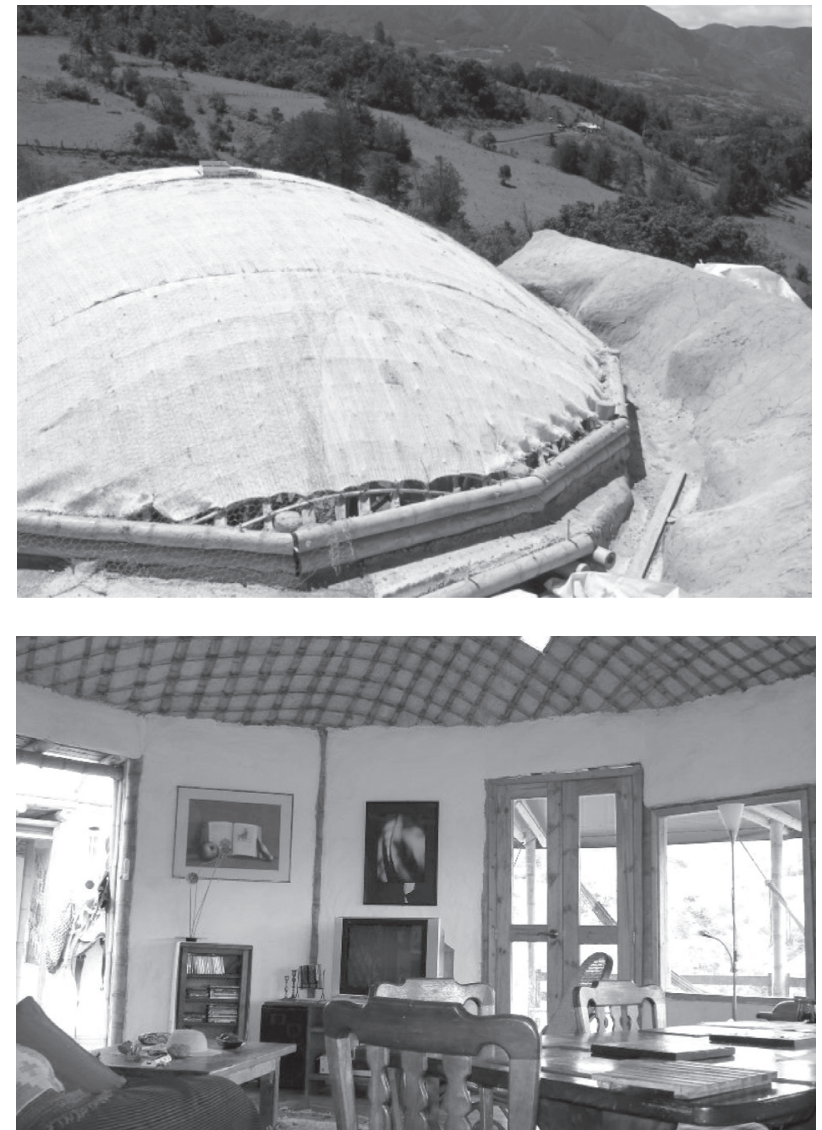

Figura 6. Colocación de costal de fique y malla de gallinero para reducir la fisuración del mortero de barro, vista exterior e interior. Fuente: propia

húmedo. Por ello, y dependiendo del tamaño de la luz, se buscan otros apoyos temporales. La capa de mortero no debe superar los $8 \mathrm{~cm}$. para evitar sobrecargar la estructura.

\section{Uso de la tierra}

La tierra es usada como material de aislamiento térmico y acústico. La función de este material, como ocurre con el bahareque tradicional para paredes, es aplicarse como envoltura; también sirve de protección, de cobijo, ya que "es como la piel, pues regula la temperatura, la humedad y el sonido" (Garzón, 2011, p. 62).

La tierra al ser un material no combustible protege las estructuras contra incendios; adicionalmente 
al usarla en cubiertas tiene una importante función de controlar los cambios climáticos y, con las formas curvas, cumple funciones bioclimáticas dependiendo del lugar, regulando el frío o el calor.

Neves (2011, p. 9) comenta que "desde la segunda mitad del siglo XIX, el uso habitual de la tierra fue cediendo inevitablemente ante la aparición de materiales de construcción industrializada, y así la tierra se quedó al margen de las obras públicas y privadas, donde comenzó a competir con el gusto de los patrones estéticos dictados por los nuevos materiales (pág. 10). y, afirma también que "los problemas ambientales causados por el hombre en el proceso de producción de bienes de consumo, así como la producción sostenible del espacio urbano, se hicieron evidentes a finales del siglo XX, lo que dio como resultado la sensibilización de la sociedad sobre la urgente necesidad de revertir esta situación" (p. 10).

Por ello técnicas como la del domocaña, cuyo desarrollo está proyectado para los nuevos tiempos, retoma materiales que como consecuencia de la crisis energética y las problemáticas ambientales están renaciendo, tales como la tierra y la guadua que empiezan a posicionarse, visibilizarse y hacer parte de una arquitectura sostenible y cercana.

La tierra para cubiertas solo se ha usado en la arquitectura en regiones secas, con muy bajo nivel de pluviosidad. Sin embargo, con la posibilidad de fusionar la técnica de cubiertas como el domocaña con otros materiales que ofrece el desarrollo industrial dentro de la óptica ecológica, la tierra se convierte en la piel que regula algunas condiciones fundamentales en la habitabilidad de la edificación y remplaza el concreto, material que tiene mayor impacto ambiental.

La tierra tiene cualidades únicas, respira y tiene mejor comportamiento ante la condensación interior; al mezclarla con otros materiales naturales es compatible en su esencia, aporta propiedades exclusivas de su naturaleza y se encuentra en todas partes a costos muy accesibles.
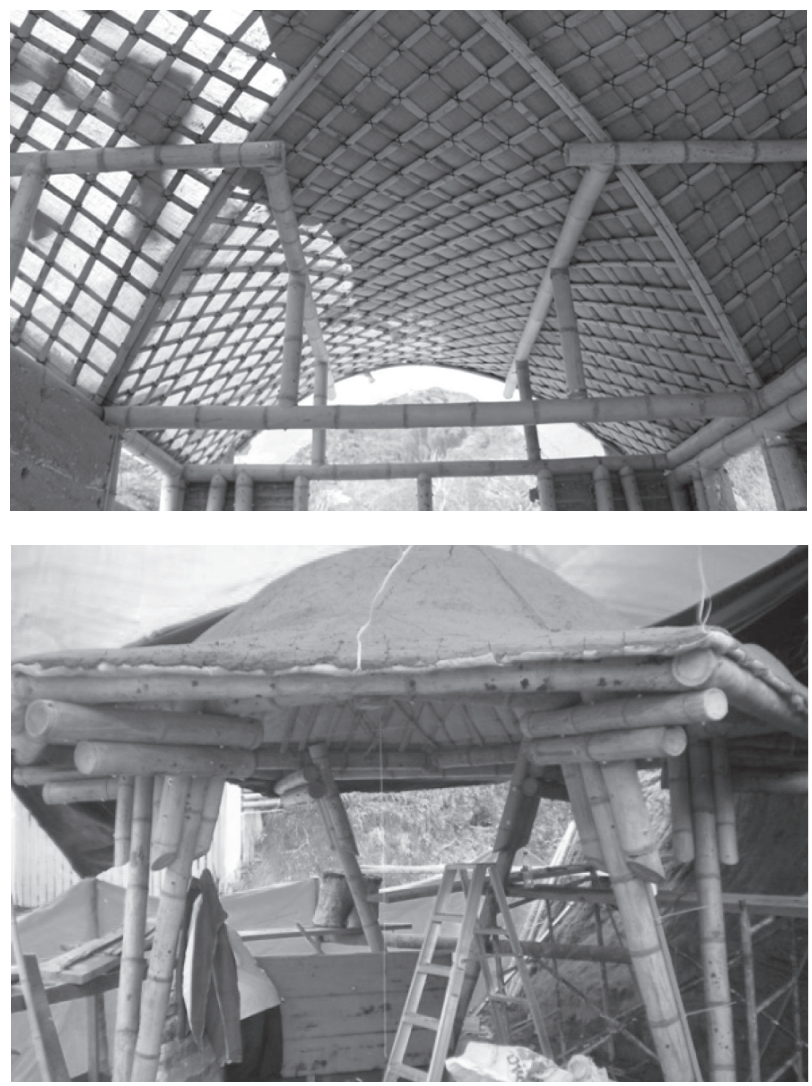

Figura 7. Revoco o empañetado de las cubiertas de domocaña en Cundinamarca y en la zona cafetera. Fuente: propia

Para elaborar el revoco se coloca sobre la tela que sirve para soportar el mortero en dos a tres capas: la primera de tierra, fibra vegetal, cal y arena; la segunda de cal, arena y cemento; y, la tercera, de arena y cemento. Éstas constituyen la base de la cubierta para la colocación del manto asfáltico impermeabilizante. La granulometría de las capas debe ir disminuyendo para llegar menos porosa a la superficie. Dependiendo del clima se puede hacer la impermeabilización incluso con materiales naturales como la cal u otros.

La impermeabilización usada en algunas de estas experiencias se ha hecho con materiales comerciales como la tela asfáltica, que está elaborada con tres materiales: cartón, asfalto y aluminio, de $1.2 \mathrm{~mm}$ a $3 \mathrm{~mm}$, o también puede aplicarse una pintura impermeabilizante con fibras, colocada en tres capas y con una membrana flexible. 
Estas impermeabilizaciones se deben colocar siguiendo las recomendaciones de los fabricantes. Es importante tener en cuenta que como estas estructuras son livianas y flexibles, los materiales de los impermeabilizantes deben tener también flexibilidad para que no se quiebren ante los movimientos que puedan absorber las estructuras.

La impermeabilización se aplica sobre la última capa delgada de mortero de cemento y arena, la cual debe estar ya seca para asegurar su durabilidad. En el caso del manto asfáltico se coloca una mano de emulsión asfáltica diluida en agua y se deja secar. Sobre esa base se instala el manto y, en las uniones y en la base, se adhiere con soplete con cuidado para evitar futuras goteras.

Es interesante explorar la posibilidad de aplicar polímeros orgánicos para la impermeabilización, ya que en países como Brasil se está ofreciendo en el mercado este tipo de productos más acordes con el cuidado ambiental y técnico de dichas cubiertas.

En el diseño de las cubiertas debe de proyectarse previamente los aleros, los detalles de unión de volúmenes y la forma de escurrimiento de las aguas lluvias; pues además de cubrir, la cubierta puede servir para la recolección y aprovechamiento de éstas, un elemento importante en los tiempos que corren. Igualmente con las formas orgánicas, las filtraciones pueden causar deterioro en la estructura por lo tanto el proyecto debe planearse integralmente $y$, en especial, los encuentros entre las cubiertas y las canales de las aguas de lluvias.

También se pueden realizar cubiertas muy económicas en espacios que por su uso, son menos exigentes en calidad térmica o acústica como parqueaderos, kioscos, lavanderías, depósitos, etc. que no requieren de un confort térmico. Para ello se puede reemplazar el costal de la superficie de la cubierta por esterilla de guadua y usarla por la textura rugosa en la parte superior, sobre la cual se puede aplicar directamente la impermeabilización con calor y soplete.

Y para finalizar como acabado para los techos, con el fin de proteger la impermeabilización, se
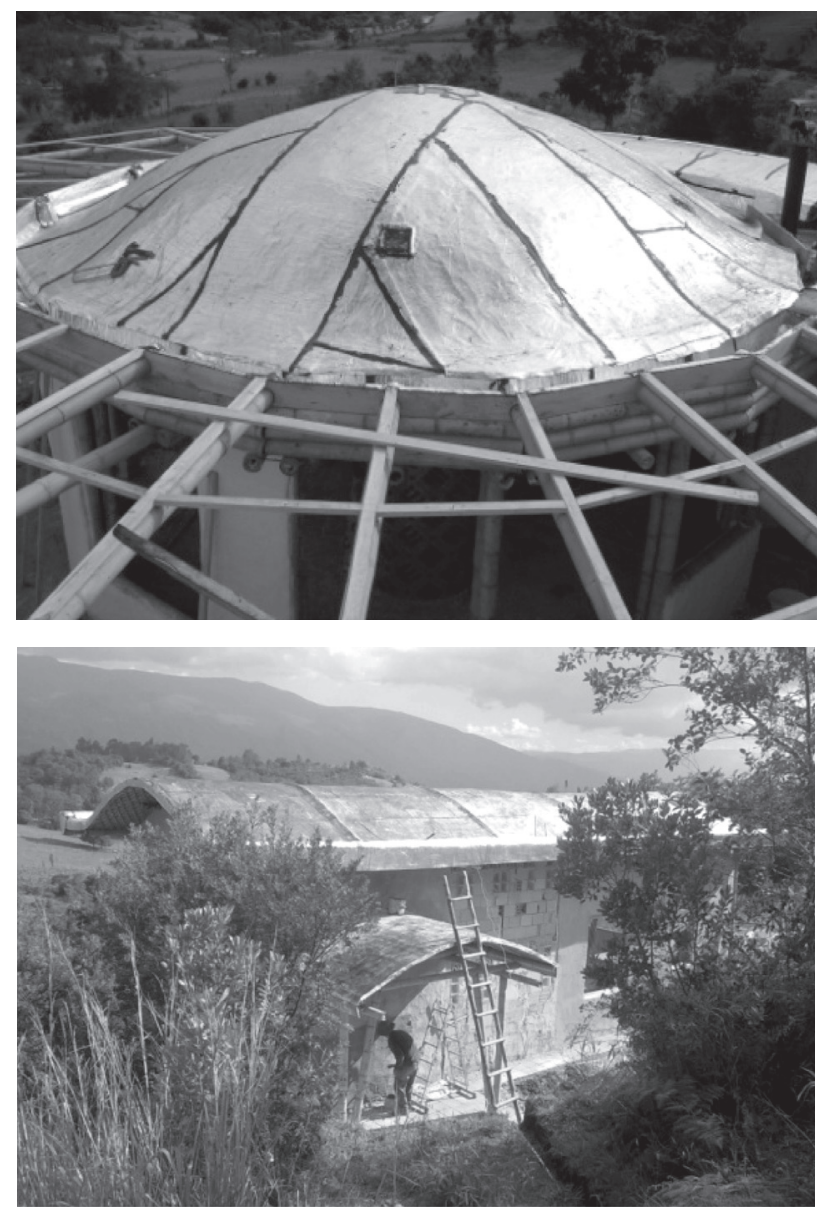

Figura 8. Impermeabilización de cubiertas con manto asfáltico y mortero. Fuente: propia

recomienda pintar con pinturas acrílicas para exteriores. La elección de la pintura es importante por la durabilidad y se recomienda mantenerla regularmente cada tres o cuatro años.

En el desarrolo de la técnica del domocaña una recomendación de los investigadores peruanos es colocar mortero por encima y por debajo de la trama de latas de guadua, con el fin de tener mayor conservación del material vegetal. En el caso de Colombia por la calidad de la guadua y para aprovechar la belleza del material así como la textura interior de la cubierta, en casi todas las experiencias se ha dejado el entramado con las latas de guadua a la vista, colocando el mortero solamente en la parte superior, brindando una estética rústica y hermosa. 

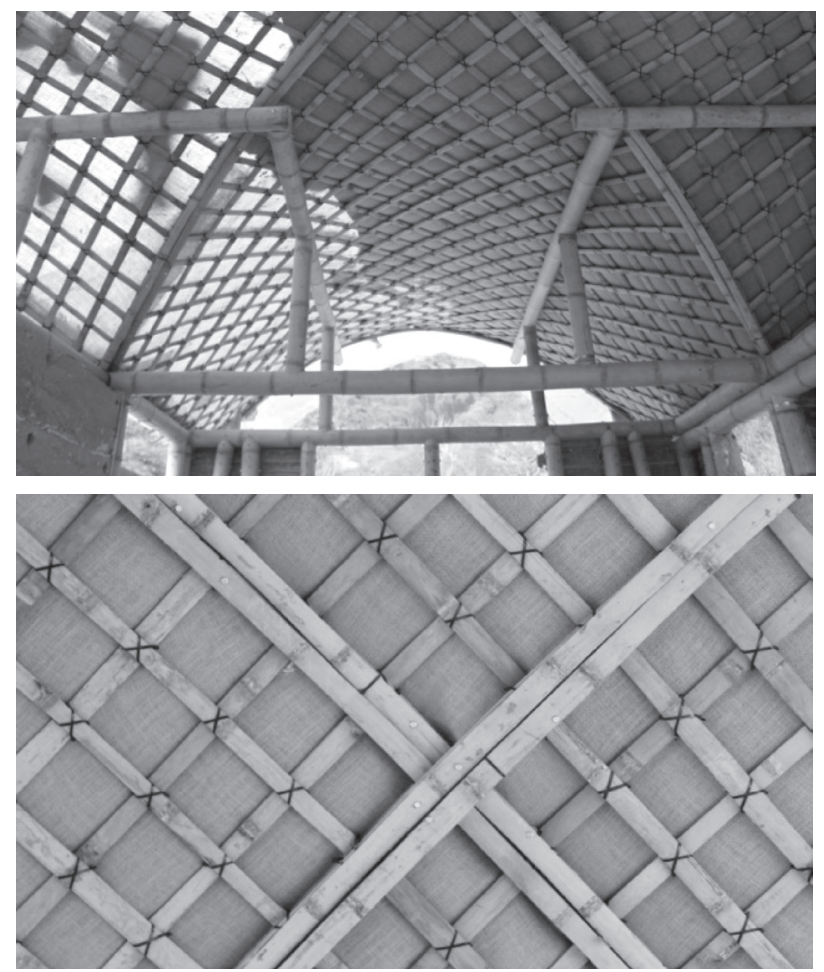

Figura 9. Vista interior y textura del domo caña en la Obra de La vega Cundinamarca. Fuente: propia

\section{Inventario de domocañas en Colombia}

La primera obra con domocaña se realizó en el año 2004 en la casa "Villa Sarita", en Villa de Leyva, Boyacá, con un diámetro de $4 \mathrm{~m}$. Posteriormente en Cochahuaira, una ecoaldea localizada en Gachantivá, Boyacá; allí desde el año 2006 se han construido dos cúpulas de 6 metros de diámetro, sobre una base poligonal de 15 lados, una bóveda curva con un ancho que crece de 2,50 a 6 metros, una bóveda de 13 metros de largo por 4 de ancho, y una bóveda de 14 metros de largo por 5 metros de ancho. También hay otra cúpula de 6 metros de diámetro en la misma vereda, una vivienda con planta en forma de cruz con dos bóvedas interceptadas de $14 \mathrm{~m}$ y una bóveda rectangular, además de otras más pequeñas en la vereda Gachantivá Viejo, en el mismo municipio.

En San Miguel de Sema, Boyacá, se construyó en el año 2007 un domocaña experimental con otra forma de bóveda, que parte de una base rectangular y es terminada en semi-cúpulas en ambos extremos.

En la zona cafetera de Santa Rosa de Cabal durante los años 2007 y 2015 se realizaron dos proyectos con talleres del domocaña; el primero en una granja ecológica dentro del marco de un taller de transferencia de bahareque y domocaña, y el segundo, en un restaurante en Guacas. Allí se comenzó con una cúpula de $3 \mathrm{~m}$ de diámetro, realizando la estrategia de un diseño con el mínimo uso de guadua en sus apoyos; se partió de la figura de un triángulo enmarcado en un hexágono con solo tres puntos de apoyo y se elaboró en el marco de un taller de transferencia tecnológica. En este lugar se construyeron otras tres bóvedas con la misma técnica. También se han realizado talleres de domocaña en varios eventos en el país desde hace 14 años, todo con el objetivo real de transferir conocimientos, colectivizar la experiencia y motivar a investigar esta técnica.

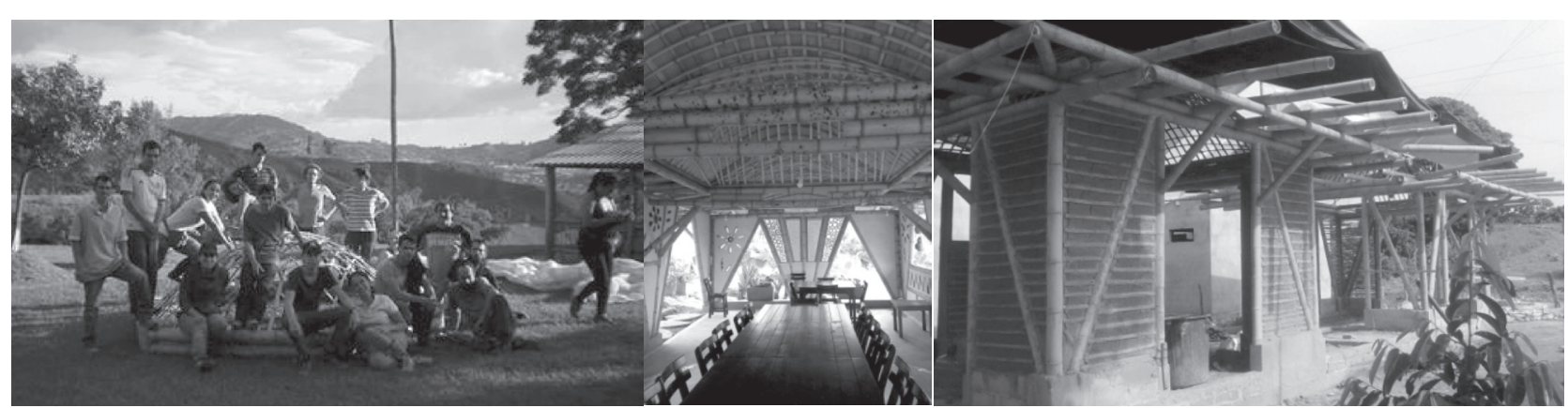

Figura 10 - Taller de transferencia tecnológica de domocaña en Santa Rosa de Cabal Risaralda y obras para un restaurante y un hotel en Palomino, Guajira. Fuente: Lucía Garzón 
En Palomino, Guajira, se realizaron cuatro cúpulas de 4 x 4 metros; y en Minca, cerca de Santa Marta, Magdalena, desde el 2017 se está construyendo la sede del teatro Cenit, con cúpulas cuadrangulares de 5,50 metros.

En el año 2018 se construyó una vivienda prefabricada de $110 \mathrm{~m} 2$ con domocañas en la Vega, Cundinamarca. En el proceso de desarrollo de esta obra participaron 40 estudiantes del SENA que realizaban un curso de construcción civil en el municipio de Villeta; todo con la mira de estimular la formación de personas conocedoras de esta técnica que puedan servir como mano de obra capacitada.

\section{Reflexiones}

En el país se producen distintas variedades de guadua con diferentes características. De ahí que algunas entidades y profesionales colombianos estén realizando investigaciones y desarrollos tecnológicos que apuntan hacia las innovaciones a partir de este recurso natural. En ese sentido un equipo de investigación sobre la madera y la guadua de la Universidad Nacional, sede Bogotá, ha desarrollado durante los últimos 10 años una laboar de indagación en torno a los aspectos críticos de este material; con ello se espera fortalecer los desarrollos tecnológicos para las cubiertas como el domocaña.

El comportamiento de las obras experimentales hechas desde hace más de una década, comprueba y garantiza la posibilidad de usar esta técnica para construcción de cubiertas económicas y ecológicas. Se reconoce que aún faltan ensayos científicos con estudios complementarios que contemplen los cuidados en el tratamiento de los materiales.

Es recomendable comenzar por proporciones pequeñas y aunque la técnica permite "arriesgarse" con luces mayores y formas variadas, están pendientes los aportes de los estudios de ingeniería que podrían tener mayor evolución, replicando lo que ocurrió con la versatilidad de formas de los cascarones de concreto. Lo anterior significa que esta técnica evolucionará siempre y cuando se incrementen las investigaciones al respecto.

La guadua técnicamente exige un conocimiento, manejo y recolección de acuerdo a las normas técnicas del Instituto Colombiano de Normas Técnicas (ICONTEC, 2006a; 2006b; 2007a; 2007b; 2008; 2014). En cualquiera de estos proyectos se recomienda el uso de la guadua y la esterilla cortada en tiempo de cuarto menguante e inmunizada con materiales inocuos para la salud humana y ambiental, como son el pentaborato de sodio, una mezcla de ácido bórico y bórax diluido en agua.

Como ya se anotó al comienzo del artículo, con este trabajo de transferencia de tecnología en la construcción de los domocañas a partir de las experiencias y reforzando esta técnica con la investigación académica y científica, se visualiza establecer los sistemas de cálculo para este tipo de cubiertas, proyectar la técnica y posteriormente crear los protocolos que aseguren la sismo-resistencia; así se puede sopesar los alcances de esta forma de construir cubiertas, proponer la creación de las normativas e incursionar en la política pública con directrices que permitan aplicar esta tecnología en vivienda rural, económica y sostenible.

La técnica del domocaña es simple y fácil: es "una técnica blanda". Las limitaciones y tareas de los que la aplican están en conocer y estudiar los materiales no convencionales que, por ser no estandarizados, exigen un conocimiento práctico de alto nivel, en especial en lo que respecta a la estructura, (garantía de la guadua en el tiempo), ya que ella es básica para una cubierta.

La posibilidad formal, tal como ocurrió con los cascarones de concreto, va a depender de la creatividad e investigación del manejo de la geometría para ofrecer nuevas ideas espaciales y estéticas. 

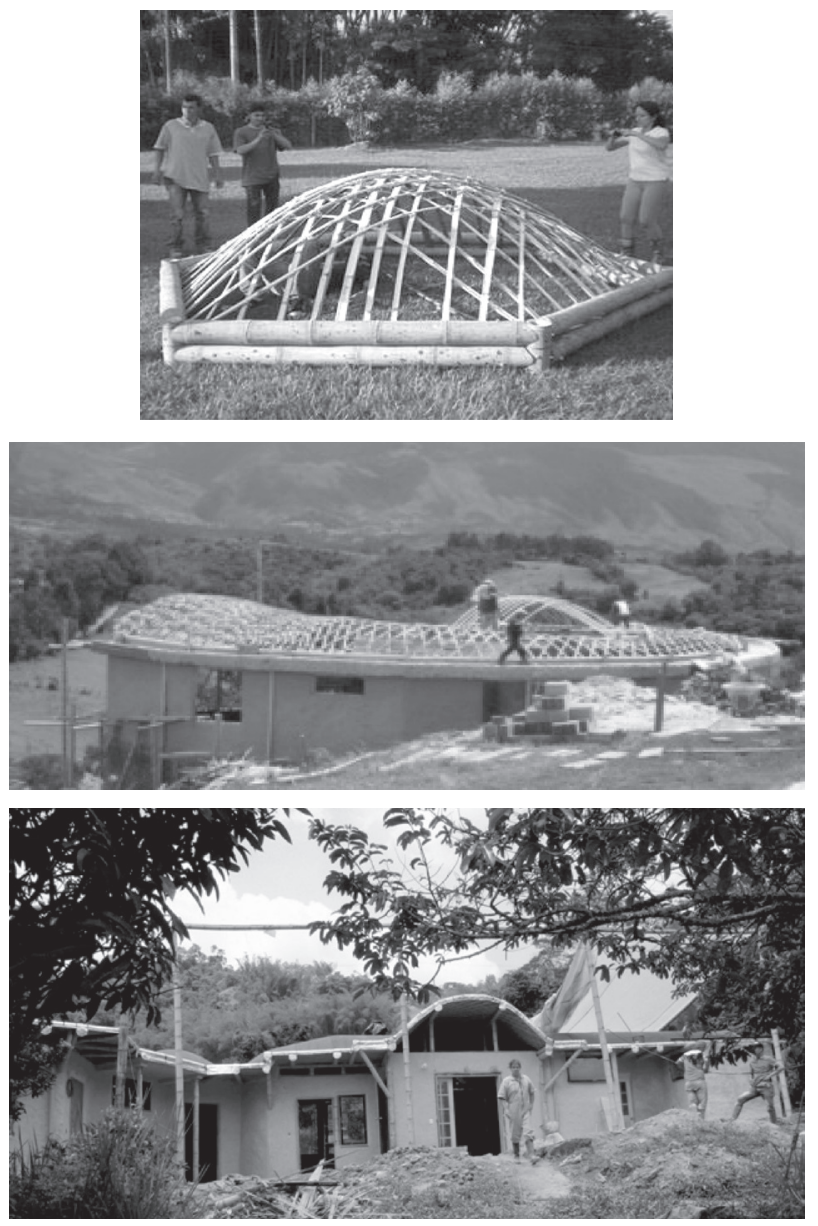

Figura 10. Forma de cubiertas con domocaña: cúpula y doble curvatura. Fuente: propia

\section{Conclusiones}

\section{- Elección de las formas, el comportamiento y las resistencias estructurales}

Las formas de las plantas arquitectónicas y la dirección del entramado o malla, determinan la resistencia de las cubiertas elaboradas con esta técnica; por la experiencia se puede decir que las plantas cuadradas con los domos o formas de cúpula, son más estables que las plantas rectangulares con las formas de bóvedas y, en el caso de las cubiertas de doble curvatura con la cúpula y la bóveda curva que rodea la planta en espiral como la de tipo amonita, la resistencia es mucho mayor que la de las bóvedas rectangulares.

\section{- Tecnología blanda y accesibilidad}

Con la experiencia lograda en varios talleres y la vivencia en obras, se ha demostrado la fácil aceptación social y la ágil transferencia de conocimientos para el aprendizaje de la técnica. De esta forma la cualificación de la mano de obra se realiza de forma rápida y ello demuestra que es un recurso accesible a la hora de masificar la técnica. También tiene poca exigencia de herramientas e incluso en la adaptabilidad de sus materiales.

\section{- Transferencia de conocimiento}

Con las experiencias obtenidas durante estos años se puede realizar un prototipo de cubierta en muy corto plazo. En talleres de dos días es posible aprender los detalles, conocer los principios y capacitarse en los aspectos fundamentales de la técnica, aunque para manejar temas del sistema constructivo integral y estructural, se requiere más tiempo. El domocaña puede ser aprendido en un breve taller. Cabe mencionar que desde Colombia ya se está exportando este método a países como Venezuela, donde se está aplicando en áreas rurales como recurso técnico, pues brinda una alternativa constructiva frente a la crisis de materiales convencionales que en este momento sufre ese país.

\section{- Investigación y sistematización de experiencias}

Sin duda se requiere continuar realizando un trabajo científico sistemático e interdisciplinario que conduzca al desarrollo homologado de esta técnica y para lograr la normativa necesaria para su aplicación en proyectos de vivienda de interés social, principalmente en zonas rurales, o en donde el contexto ambiental sea pertinente. En el caso de Colombia con los resultados obtenidos en más de diez años de experimentos y con más de 20 domocañas construidos, se puede afirmar que esta técnica es una alternativa de cubierta eficaz, versátil, lógica y sostenible, desde lo arquitectónico y lo estructural. Es indispensable, incluir los aportes necesarios desde la ingeniería, los 
ensayos de laboratorio y estudios especialmente en los aspectos estructurales y de la resistencia, así como de los procedimientos de uso, tratamiento, producción y mantenimiento de la guadua.

\section{- La guadua como potencial}

La técnica de domocaña en Colombia amplía y demuestra el alto potencial como sistema de cobertura, al ofrecer versatilidad de usos y aplicaciones de la guadua en la construcción y la exploración de otras especies de bambusas como material para cubiertas. En nuestro país el desarrollo tecnológicoen este campo es más ágil que en otras regiones del mundo debido a los avances en la investigación de las propiedades de la guadua Angustifolia Kunth. Por ello hoy es un material que ha logrado una aceptación social y científica pues, además de posicionarse en el mercado de la construcción, es una riqueza natural de gran diversidad que se encuentra en múltiples ecosistemas.

\section{Referencias bibliográficas}

Barrionuevo R. I. (2011). Investigación tecnológica aplicada: Domocaña. Informes de la Construcción Vol. 63, 523, pp. 51-58. Julio-septiembre 2011 ISSN: 0020-0883.

Garzón, L. (2011). Técnicas de construcción con tierra / Célia Neves y Obede Borges Faria, organizadores. -- Bauru : FEBUNESP / PROTERRA, 2011. 79 p. il.
Lorenzo, P. Massuch, H. Leyva, L. Barrionuevo. R y otros (2002). Un techo para vivir. Capítulo 2. Proyecto xiv.5 Con techo y programa 10x10. Acciones Cúpula de ferrocemento domocaña, pp. 29, 32, 59, 91, 97, 105, 254-257

Neves, C. (2011). Introducción. En: Neves, C.; Faria, O. B. (org.). Técnicas de construcción con tierra. Bauru: FEBUNESP / PROTERRA. Disponible en: http://www.redproterra.org

FUNDASAL (2006). Tecnodemo 2: Domocañas, cúpula de tierra. Talleres de transferencia de tecnología. En: Seminario Internacional Construcción con Tierra, San Salvador, El Salvador: FUNDASAL. p. 123.

ICONTEC, (2006a). NTC 5405 Propagación vegetativa de Guadua angustifolia Kunth".

ICONTEC, (2006b). NTC 5407 Uniones para estructuras construidas en Guadua angustifolia Kunth.

ICONTEC, (2007a). NTC 5301 Preservacion y secado del culmo de guadua angustifolia kunt.

ICONTEC, (2007b). NTC 5525 Metodos de ensayo para determinar las propiedades fisicas y mecanicas de la guadua angustifolia kunt.

ICONTEC, (2008). NTC 5300 Cosecha y pos cosecha de los culmos de Guadua angustifolia Kunth.

ICONTEC, (2014). NTC 6100 Criterios ambientales para productos de primero y segundo grado de tranformación de guadua angustifolia Kunt. 